\title{
Cardiovascular imaging in the acute phase of coronavirus disease 2019 (COVID-19)
}

\author{
Wioletta Sacharczuk \\ 2nd Department of Cardiology, Poznan \\ University of Medical Sciences, Poland \\ (iD) https://orcid.org/0000-0001-6586-7121 \\ Corresponding author: wioletta.sacharczuk@wp.pl

\section{Rafał Dankowski} \\ 2nd Department of Cardiology, Poznan \\ University of Medical Sciences, Poland \\ (D) https://orcid.org/0000-0003-0843-5378 \\ Anna Marciniak \\ St. George's, University of London
(iD) https://orcid.org/0000-0001-6590-1780
}

Anna Szałek-Goralewska

2nd Department of Cardiology, Poznan

University of Medical Sciences, Poland

(iD) https://orcid.org/0000-0002-2240-9531

\section{Andrzej Szyszka}

2nd Department of Cardiology, Poznan

University of Medical Sciences, Poland

https://orcid.org/0000-0003-0471-7001
DOI: https://doi.org/10.20883/medical.e532

Keywords: SARS COV-2, echocardiography, cardiac magnetic resonance, computed tomography, multimodality imaging, COVID-19

Published: 2021-09-28

How to Cite: Sacharczuk W, Dankowski R, Marciniak A Szałek- Goralewska A, Szyszka A. Cardiovascular Imaging in the Acute Phase of Coronavirus Disease 2019 (COVID19). Journal of Medical Science. 2021 Sep. 28;90(3):e532 doi:10.20883/medical.e532

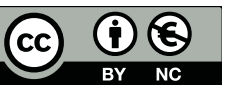

(C) 2021 by the author(s). This is an open access article distributed under the terms and conditions of the Creative Commons Attribution (CC BY-NC) licencse. Published by Poznan University of Medical Sciences

\begin{abstract}
The coronavirus disease 2019 (COVID-19) has become the most critical healthcare issue worldwide since the pandemic was announced in March 2020. Although respiratory symptoms remain the critical characteristic feature of COVID-19 (with acute respiratory syndrome as the leading cause of mortality), the disease also affects other organs. In fact, the involvement of the cardiovascular system during COVID-19 may include acute coronary symptoms, acute heart failure and myocarditis, arrhythmias, cardiac tamponade, pulmonary embolism, and right ventricular failure due to a high-pressure mechanical ventilation. It is vital to note that all of the abovementioned disorders require specific, pandemic-adapted imaging algorithms.

This brief review aims to discuss different cardiac imaging modalities to demonstrate their effectiveness in managing patients in the acute phase of COVID-19.
\end{abstract}

\section{Introduction}

Cardiovascular involvement among patients infected with severe acute respiratory syndrome coronavirus 2 (SARS-CoV-2) is common and significantly deteriorates the prognosis [1].

Hence, it requires quick diagnostic diagnosis and immediate treatment. In addition, several cardiac complications have been reported in the acute phase of COVID-19, such as myocarditis [2], pericarditis [3], acute myocardial infarction [4], stress-induced cardiomyopathy [5], cardiac tamponade [6], and / or right ventricular failure due to a high-pressure mechanical ventilation [7]. All these clinical situations require imaging techniques as a primary diagnostic tool.

Due to the sanitary regime during the COVID-19 pandemic, the availability of diagnostics may be 
limited. Consequently, new or modified diagnostic pathways have recently been developed [8].

In this short review, we address the relevant issues concerning cardiovascular and pulmonary imaging, and share our experience from the COVID-19 ward.

\section{Computed tomography}

Computed tomography (CT) is the primary imaging tool in COVID-19 patients. In fact, high-resolution CT (HRCT) allows for the assessment of pulmonary involvement: pneumonia and pulmonary fibrosis. Typical images include bilateral, multifocal, multi-lane frosted glass with or without sub-segment consolidation, or a "crazy paving" pattern in the circumferential distribution. Results are usually presented as a percentage of infiltrating pulmonary parenchyma (Figure 1).
Furthermore, CT also plays a crucial role in diagnosing pulmonary embolism (PE). The prevalence of $\mathrm{PE}$ at the time of hospital admission for COVID-19 reached $14.2 \%$, and further increased in the course of hospitalization [8]. By means of CT pulmonary angiography (CTPA) it is possible to confirm blood clots in the pulmonary arteries, or in the right heart chambers (Figure 2). The calculation of the right ventricle enlargement is a simple method to assess ventricular overload [9].

CT may constitute a helpful tool in the diagnosis of cardiovascular diseases and their complications, as on the basis of $\mathrm{CT}$ it is possible to exclude coronary artery disease (CAD) before further diagnosis [10], or before diagnosing an aortic pathology in patients with chest pain. Another issue is atrial fibrillation, the most common type of arrhythmia in COVID-19, observed in $17 \%$ of hospitalized patients. CT successfully replaces transoesophageal echocardiography in thrombus detection prior to cardioversion [11].

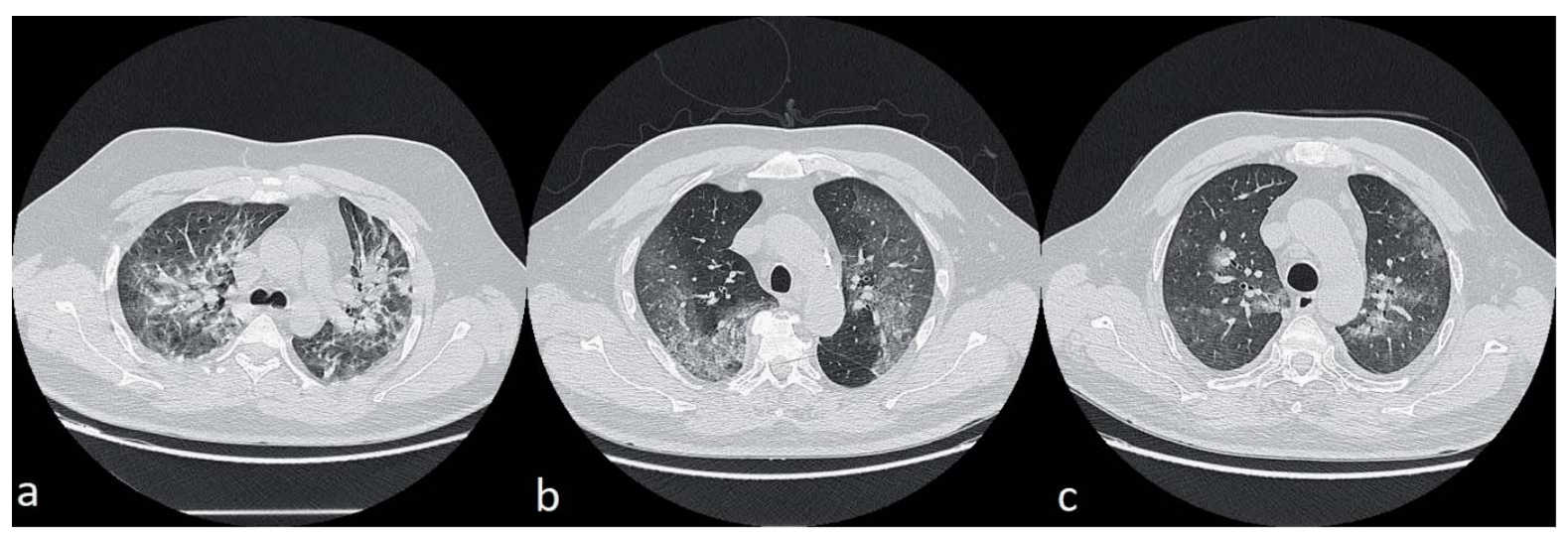

Figure 1. High-resolution CT (HRCT) presenting a percentage of pulmonary infiltration during SARS-COV 2 infection: (a) large involvement - more than $80 \%$ of infiltration, (b) $60 \%$ infiltration, (c) about $30 \%$ infiltration

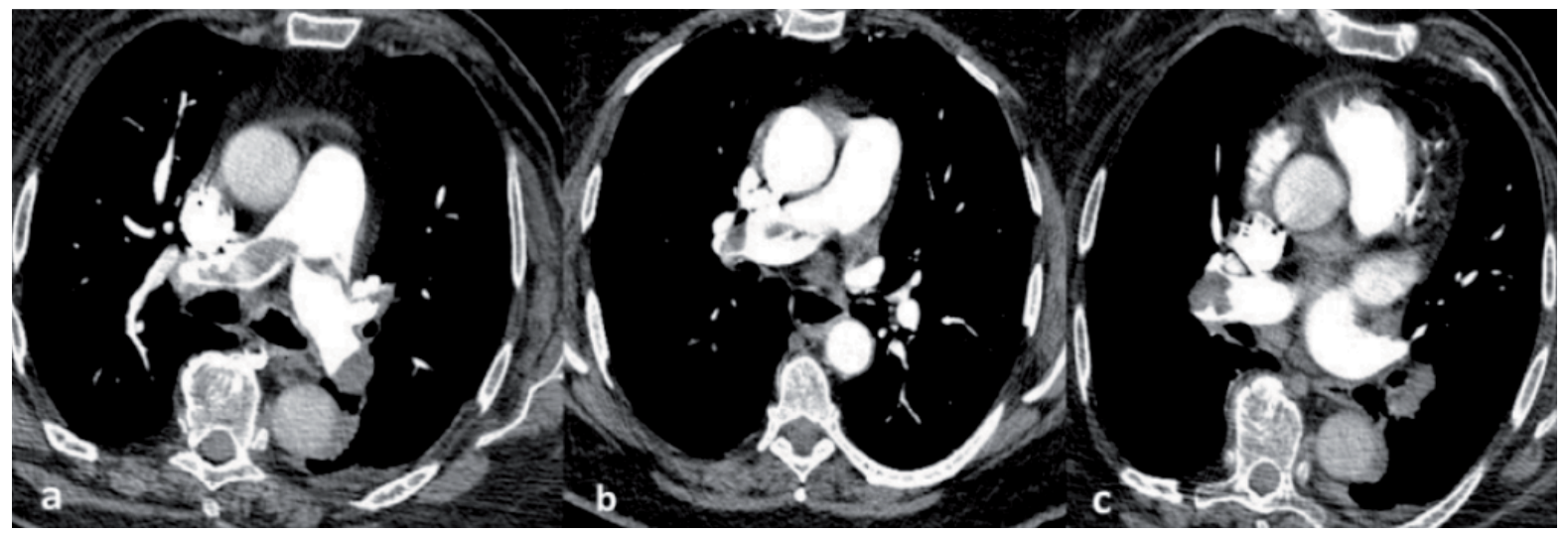

Figure 2. Pulmonary embolism in the acute phase of COVID-19: (a) large embolus in the pulmonary trunk and pulmonary arteries, (b) small embolus in the right pulmonary artery, (c) embolus in the proximal part of the right low lobe artery 
Concluding, CT in COVID-19 patients enables the application of "the triple rule-out principle" concerning all the aforementioned conditions (Table 1).

Serious limitations in the use of CT technique include transporting the patient to the radiology department under the appropriate sanitary regime and sanitation processes.

\section{Echocardiography}

Although echocardiography is recommended as the first-line imaging tool for most cardiac conditions, it should not be routinely ordered in patients with COVID-19, particularly when no clinical benefits are expected. Transoesophageal echocardiography, which is an aerosol-generating procedure, should ideally be performed after the acute phase of COVID-19, due to an increased risk of virus transmission [12]. The primary echocardiographic modality in the management of COVID-19 patients is a point-of-care cardiac ultrasound (POCUS). Pocket size devices, such as a hand-held or a laptop-based scanner, are recommended, due to the speed of image availability, as well as bedside interpretation which allows reducing the scanning time and affects immediate patients' management. Easy transportability and a more efficient disinfection play a vital role in comparison to large machines [13]. An echocardiogram targeted at a specific clinical problem is referred to as a focused cardiac ultrasound (FoCUS), and its main goals are presented in Table 2, whereas typical echocardiographic images are demonstrated in Figure $\mathbf{3}$.

Careful evaluation of the right ventricle (RV) may be crucial in mechanically ventilated patients. Echocardiographers should pay particular attention to high positive end-expiratory pressures (PEEP) - induced cardiopulmonary overload, resulting in the symptoms of acute cor pulmonale. In fact, a dilated right ventricle with a flattened interventricular septum with a basal RV to LV ratio $>1.0$ are the most specific echocardiographic markers identifying this process [14]. Moreover, McConnell's sign, short RV outflow Doppler acceleration time, and high tricuspid regurgitation pressure gradient may indicate the presence of embolic material in pulmonary arteries $[13,15]$. In addition, POCUS may also play an essential role in the PE diagnosis in COVID-19 patients with contraindications for CT angiography (e.g. pregnant women, allergy to contrast media).

Table 1. "Triple rule-out" CT Angiography Objectives in COVID-19 patients presenting the acute chest pain

\begin{tabular}{lc}
\multicolumn{1}{c}{ The anatomical structure } & Conditions which can be excluded by CT \\
\hline Thoracic aorta & Aortic dissection \\
\hline Coronary arteries & $\mathrm{CAD}^{\mathrm{a}}$ \\
\hline Pulmonary arteries & $\mathrm{PE}^{\mathrm{b}}$ \\
\hline
\end{tabular}

Abbreviations: (a) coronary artery disease; (b) pulmonary embolism

Table 2. The focused cardiac ultrasound (FoCUS) parameters important in COVID-19 patients

\begin{tabular}{lcc}
\multicolumn{1}{c}{ Characteristics } & Left Ventricle & Right Ventricle \\
\hline Global Function & LVEF $^{\mathrm{a}}$ & $\begin{array}{c}\text { RVFAC }^{\mathrm{b}} \\
\text { TAPSE }^{\mathrm{c}}\end{array}$ \\
\hline $\begin{array}{l}\text { Regional Contraction } \\
\text { Abnormalities }\end{array}$ & Hypo-/akinetic regions & Hypo-/akinetic regions \\
\hline Diastolic Function & ABS $^{\mathrm{d}}$ & RVEDd $^{\mathrm{f}}$ \\
\hline Valves assessment & LVEDd $^{\mathrm{e}}$ & $\mathrm{TRP}^{\mathrm{g}}$ \\
\hline Others & & \\
\hline
\end{tabular}

Abbreviations: (a) LVEEF - left ventricular ejection fraction; (b) RVFAC - right ventricular fractional area change, (c) TAPSE - tricuspid annular plane systolic excursion; (d) ABS - apical ballooning syndrome (typical in the Tako-Tsubo syndrome); (e) LVEDd - left ventricular end-diastolic dimension; (f) RVEDd - right ventricular end-diastolic dimension; (g) TRP - tricuspid regurgitation pressure gradient 

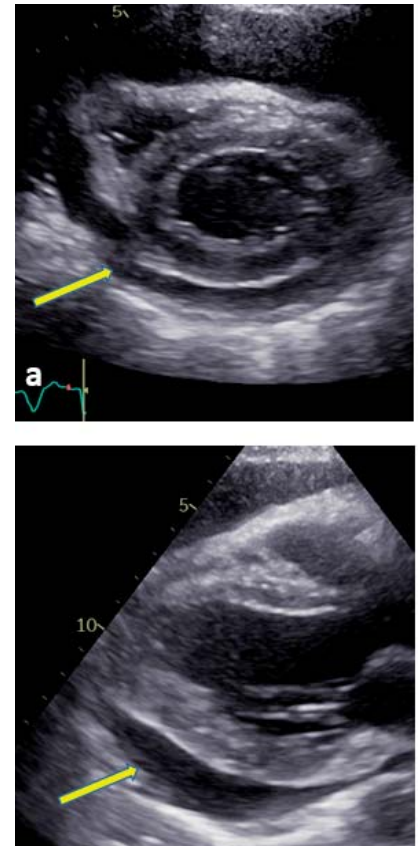

b
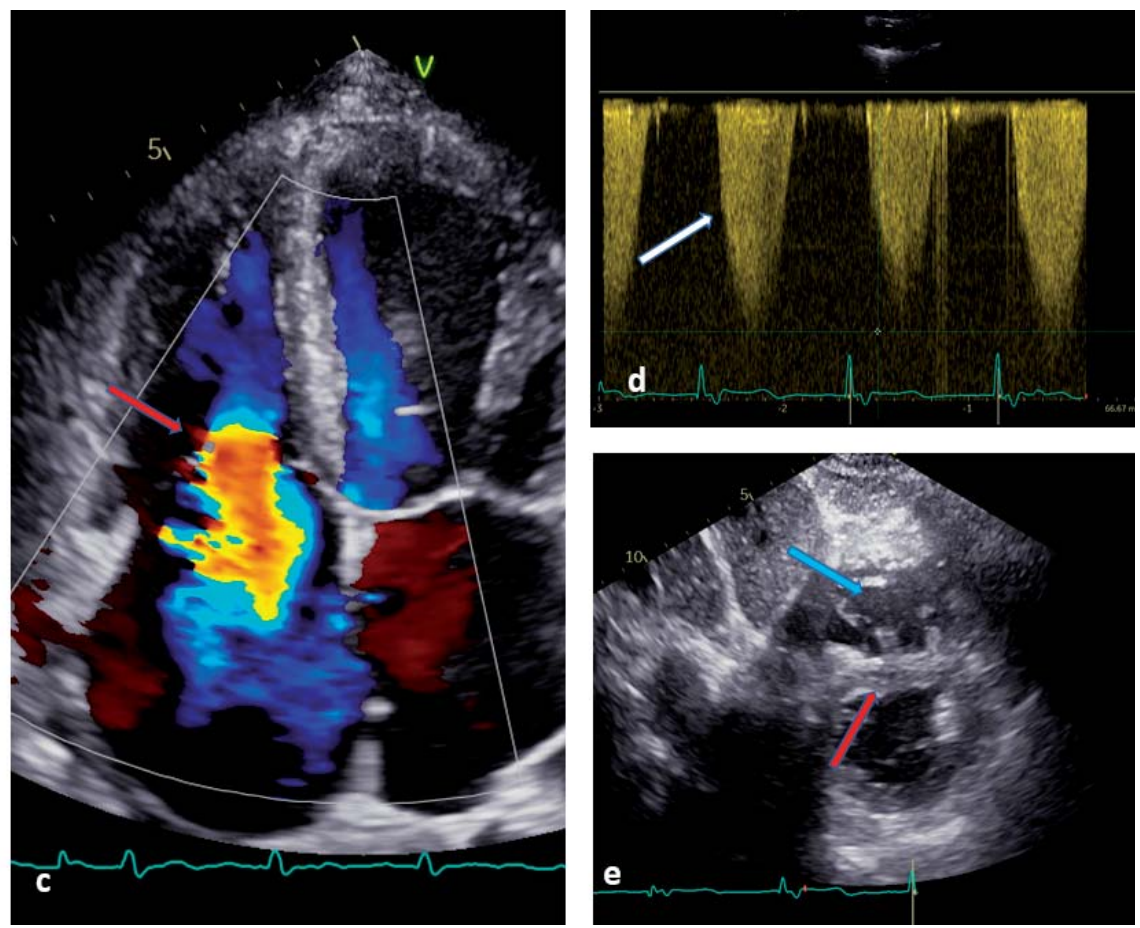

Figure 3. FoCUS images: (a) Parasternal Short Axis View (PSAX) with pericardial effusion - yellow arrow, (b) Parasternal Long Axis View (PLAX) with pericardial effusion - yellow arrow, (c) Apical 4-chamber View (4CH) with severe tricuspid regurgitation - red arrow, (d) Continuous-wave Doppler performing tricuspid regurgitation - white arrow, (e) Dilated right ventricle - blue arrow with a "flattening" of the interventricular septum as a result of high pressure in the right ventricle - red arrow

\section{Lung point-of-care cardiac ultrasound (lung POCUS)}

"Light beam" artifacts caused by subpleural consolidation can be early detected in COVID-19. They correspond to the "ground-glass" haze observed in CT imaging of COVID-19 pneumonia. The identification of the $B$ lines in the POCUS examination may constitute an additional diagnostic value in tomography imaging $[16,17]$.
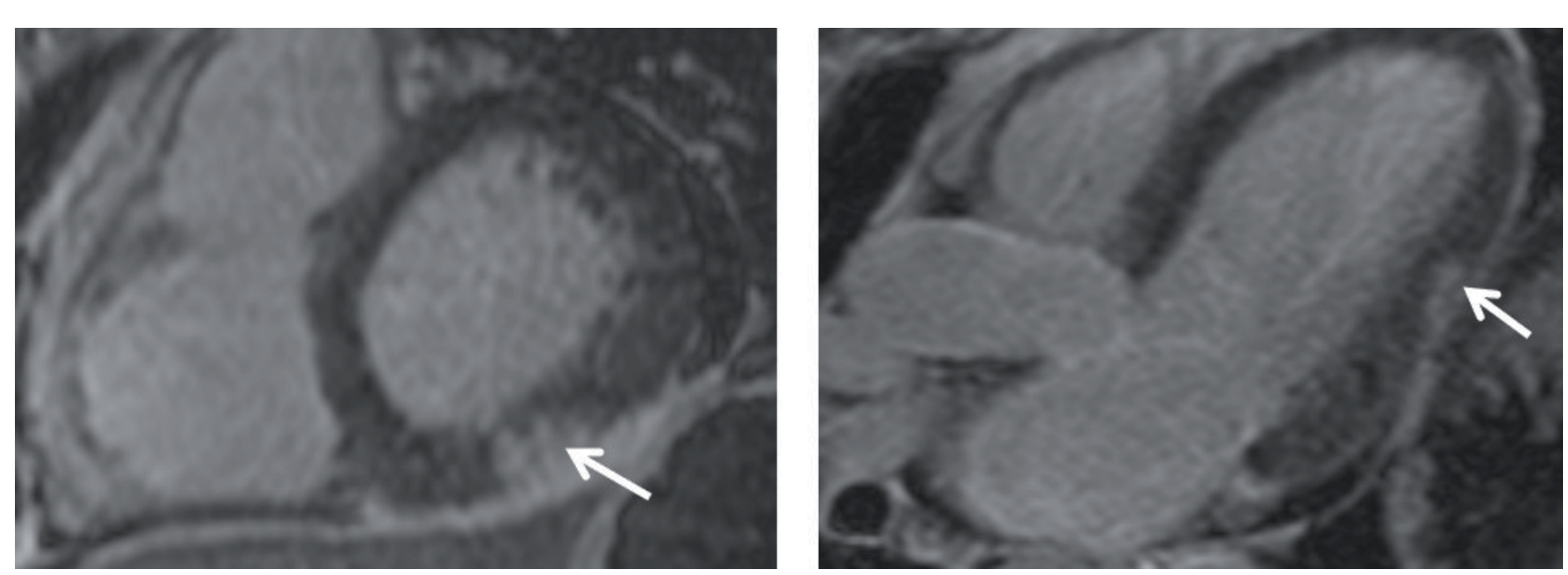

Figure 4. An example of a patient suffering from COVID-19, and an associated troponin increase with a subepicardial, myocarditis-pattern, late gadolinium enhancement in the mid-inferior wall extending to the inferolateral segment (white arrows)

\section{Cardiac magnetic resonance (CMR)}

CMR is currently considered the gold standard for tissue imaging in the assessment of myocardial oedema and fibrosis by late gadolinium enhancement [18]. It is vital to bear in mind that in patients with COVID-19 it allows to distinguish the non-ischemic myocarditis type (Figure 4) from the ischemic mechanism of a myocardial injury [19]. Furthermore, when coupled with T1 and T2 
mapping, CMR improves the diagnostic accuracy and identifies both acute and chronic changes of myocardial inflammation [20]. Interestingly, the most recent multicentre study has demonstrated that patients surviving severe COVID-19 who show elevated troponin levels and ongoing localized inflammation represent an emerging issue of clinical relevance. In patients with a severe COVID-19 course and a positive troponin assay, the evidence of residual inflammation on early CMR may play a role in the pathophysiology of dilated cardiomyopathy [19]. Nevertheless, a cost and availability issue for this technique may be a limiting factor for this modality.

\section{Conclusions}

The worldwide spread of SARS-CoV-2 disease revealed numerous weaknesses of healthcare systems, and changed our diagnostic pathways in patients suffering from COVID-19. Additionally, it also motivated the experts to create unique recommendations, algorithms, and practical guidelines to diagnose and treat infected patients, one of which is the employment of short protocols of cardiac imaging, which should be routinely applied due to the epidemic risk.

In our experience, CT may be selected as a "one-stop-shop" imaging method in COVID-19 patients, as it allows for obtaining various parameters in a single screening. Moreover, mobile devices, as well as POCUS and FoCUS protocols, should be the preferred choice when performing echocardiography, whereas CMR should be performed in troponin-positive patients, if available.

\section{Acknowledgements}

\section{Conflict of interest statement}

The authors declare no conflict of interest.

\section{Funding sources}

There are no sources of funding to declare.

\section{References}

1. Nishiga M, Wang DW, Han Y, Lewis DB, Wu JC. COVID-19 and cardiovascular disease: from basic mechanisms to clinical perspectives. Nat Rev Cardiol. 2020 Sep;17(9):543-58.

2. Zeng J-H, Liu Y-X, Yuan J, Wang F-X, Wu W-B, Li J-X, et al. First case of COVID-19 complicated with fulmi- nant myocarditis: a case report and insights. Infection. 2020 0ct;48(5):773-7.

3. Kumar R, Kumar J, Daly C, Edroos SA. Acute pericarditis as a primary presentation of COVID-19. BMJ Case Rep. 2020 Aug 18;13(8):e237617.

4. Capaccione KM, Leb JS, D'souza B, Utukuri P, Salvatore MM. Acute myocardial infarction secondary to COVID-19 infection: A case report and review of the literature. Clin Imaging. 2021 Apr;72:178-82.

5. Gomez JMD, Nair G, Nanavaty P, Rao A, Marinescu K Suboc T. COVID-19-associated takotsubo cardiomyopathy. BMJ Case Rep. 2020 Dec 12;13(12):e236811.

6. Hua A, O'Gallagher K, Sado D, Byrne J. Life-threatening cardiac tamponade complicating myo-pericarditis in COVID-19. Eur Heart J. 2020 Jun 7;41(22):2130.

7. García-Cruz E, Manzur-Sandoval D, Baeza-Herrera LA, Díaz-Méndez A, López-Zamora A, GonzálezRuiz $F$, et al. Acute right ventricular failure in COVID-19 infection: A case series. J Cardiol Cases. 2021 Jul;24(1):45-8.

8. Jevnikar M, Sanchez 0 , Chocron R, Andronikof M, Raphael M, Meyrignac 0 , et al. Prevalence of pulmonary embolism in patients with COVID 19 at the time of hospital admission. Eur Respir J. 2021 Mar 10;2100116.

9. Dupont MVM, Drăgean CA, Coche EE. Right ventricle function assessment by MDCT. AJR Am J Roentgenol. 2011 Jan;196(1):77-86.

10. Pontone G, Scafuri S, Mancini ME, Agalbato C, Guglielmo M, Baggiano A, et al. Role of computed tomography in COVID-19. J Cardiovasc Comput Tomogr. 2021 Feb;15(1):27-36.

11. Romero J, Husain SA, Kelesidis I, Sanz J, Medina HM, Garcia MJ. Detection of left atrial appendage thrombus by cardiac computed tomography in patients with atrial fibrillation: a meta-analysis. Circ Cardiovasc Imaging. 2013 Mar 1;6(2):185-94.

12. Skulstad H, Cosyns B, Popescu BA, Galderisi M, Salvo GD, Donal E, et al. COVID-19 pandemic and cardiac imaging: EACVI recommendations on precautions, indications, prioritization, and protection for patients and healthcare personnel. Eur Heart J Cardiovasc Imaging. 2020 Jun 1;21(6):592-8.

13. Yau $\mathrm{O}$, Gin K, Luong C, Jue J, Abolmaesumi P, Tsang $\mathrm{M}$, et al. Point-of-care ultrasound in the COVID-19 era: A scoping review. Echocardiogr Mt Kisco N. 2021 Feb;38(2):329-42.

14. Konstantinides SV, Meyer G, Becattini C, Bueno H, Geersing G-J, Harjola V-P, et al. 2019 ESC Guidelines for the diagnosis and management of acute pulmonary embolism developed in collaboration with the European Respiratory Society (ERS). Eur Heart J. 2020 Jan 21;41(4):543-603.

15. Kulkarni S, Down B, Jha S. Point-of-care lung ultrasound in intensive care during the COVID-19 pandemic. Clin Radiol. 2020 Sep;75(9):710.e1-710.e4.

16. Lomoro P, Verde F, Zerboni F, Simonetti I, Borghi C, Fachinetti $C$, et al. COVID-19 pneumonia manifestations at the admission on chest ultrasound, radiographs, and CT: single-center study and comprehensive radiologic literature review. Eur J Radiol Open. 2020;7:100231. 
17. Volpicelli G, Gargani L. Sonographic signs and patterns of COVID-19 pneumonia. Ultrasound J. 2020 Apr 21;12(1):22.

18. Karamitsos TD, Arvanitaki A, Karvounis $H$, Neubauer S, Ferreira VM. Myocardial Tissue Characterization and Fibrosis by Imaging. JACC Cardiovasc Imaging. 2020 May;13(5):1221-34.

19. Kotecha T, Knight DS, Razvi Y, Kumar K, Vimalesvaran $\mathrm{K}$, Thornton $\mathrm{G}$, et al. Patterns of myocardial inju- ry in recovered troponin-positive COVID-19 patients assessed by cardiovascular magnetic resonance. Eur Heart J. 2021 May 14;42(19):1866-78.

20. Sanghvi SK, Schwarzman LS, Nazir NT. Cardiac MRI and Myocardial Injury in COVID-19: Diagnosis, Risk Stratification and Prognosis. Diagn Basel Switz. 2021 Jan 15;11(1):130. 